\title{
Color matching functions of ten Japanese observers
}

\author{
Kanji KATORI* and Masahiro FUWA*
}

\begin{abstract}
Color matching properties of Japanese observers were examined by using a new trichromator equipped with numerical control devices. The additivity law of the color matching equation was confirmed to hold good for both of the $2^{\circ}$ and $10^{\circ}$ fields, but it was suggested that the color matching functions might be affected by the chromatic adaptation to the color stimulus pre-exposed in the visual field. Two sets of the $10^{\circ}$ color matching functions were obtained by ten Japanese observers. One of them was determined by the method of constant stimuli and the other by the method of adjustment. There were no appreciable differences between the two sets of the ETL $10^{\circ}$ color matching functions, but both of them differred a little from the data of the CIE 1964 supplementary standard colorimetric observer in the region of long wavelengths.
\end{abstract}

\section{Introduction}

Various studies have been reported on the color matching functions. Two sets of the CIE color matching functions were recommended by the CIE on the basis of some data obtained by those studies ${ }^{2) \sim 5)}$ : for the small visual field in 1931 and for the large field in 1964. Those data, however, were determined by the method of adjustment and most of observers were Westerns.

In our present study, color matching properties of Japanese observers were examined at Electrotechnical Laboratory (ETL) by using a new trichromator equipped with numerical control devices. First, the additivity of the color matching functions for the $2^{\circ}$ and $10^{\circ}$ visual fields was examined by the method of adjustment. Then, the effect of chromatic adaptation on the color matching functions was analysed by the method of constant stimuli. Finally, by both the method of constant stimuli and the method of adjustment, two sets of the ETL $10^{\circ}$ color matching functions were determined on the basis of the data for ten Japanese observers, and compared with the CIE 1964 supplementary standard colorimetric observer.

\footnotetext{
* Applied Optics Section, Electrotechnical Laboratory, 1-1-4, Umezono, Salkura-mura, Niihari-gun, Ibaraki, 305 Japan

This work was published previously in Japanese ${ }^{1}$, and presented partly at the 1.9th CIE session, Kyoto, Japan, August 21-28, 1979.
}

\section{Methods and procedures}

\subsection{Apparafus}

The instrument used in this study was a Stiles type trichromator constructed at ETL after the trichromator of National Physical Laboratory, England ${ }^{6)}$. This trichromator was newly equipped with a numerical control system and also with an optical system for presentation of chromatic adaptation light. The optical system of the ETL trichromator was composed of three double monochromators with cancelling dispersion forming the same three-tier structure as that of the NPL trichromator, as described in the previous paper ${ }^{7}$.

All the experimental procedures were performed automatically according to the numerical commands programmed on paper tapes: adjustment of the stimulus intensities by optical wedges and neutral density filters, selection of the wavelengths for test stimuli and desaturating reference stimuli, presentation of the test color stimuli and chromatic adaptation light, print out of the experimental conditions and the results of categolical judgement, and so on. All the color matching experiments were performed under the Maxwellian viewing condition. The instrumental reference stimuli $R, G$ and $B$ were the monochromatic radiations of wavelengths 670,530 and $460 \mathrm{~nm}$, respectively. Pattern of the visual field presented to the observer was formed by a circular bipartite field of $10^{\circ}$ in diameter divided horizontally and a surrounding field extending to an outer diameter of $14^{\circ}$. 


\subsection{Preliminary experiments}

(a) In order to test the Grassmann's laws on the additive color mixture, color matching experiments by the method of adjustment were performed for the $2^{\circ}$ and $10^{\circ}$ visual fields by changing the color mixture ratios of the test spectral stimulus to the desaturating reference stimulus, the luminance of the visual field being kept constant at the level of about 400 trolands. The observers were four males with normal color vision, ranging in age between 25 and 37 years.

(b) It was examined by using a categorical judgement method whether the color match of a metameric pair for the $10^{\circ}$ field persisted or not after the preadaptation by various colored lights, and the effect of chromatic adaptation on the color matching functions was investigated by applying the variance analysis method to the two-way layout of categorical values which had been obtained for the four pairs of color match under each of four kinds of chromatic adaptation lights. The observers used were ten males with normal color vision, ranging in age between 18 and 41 years.

\subsection{Main experiments}

Two sets of $10^{\circ}$ field color matching functions were measured for ten observers by two different methods, that is, the method of constant stimuli (abbreviated as MCS) with preadaptation by white light (the CIE standard source $C$ ) and the method of adjustment (abbreviated as MA). The observers were two females and eight males, ranging in age between 24 and 44 years. The observing conditions and instrumental parameters were the same as those described in the previous paper ${ }^{7)}$. 34 test stimuli in the wavelength range from 395 to $740 \mathrm{~nm}$ were used for the color matching experiments. Field intensities were kept constant at the level of about 200 trd, except both the ends of visible range.

Color matching experiments were performed as follows:

(a) By performing the experiments by MA ten times with one observer (K.K.) who was experienced in these experiments, the standard deviations $\sigma_{R}(\lambda), \sigma_{G}(\lambda), \sigma_{B}(\sigma)$ of the spectral tri-stimulus values at the wavelength $\lambda(\mathrm{nm})$ were determined.

(b) The mean values $\bar{R}(\lambda), \bar{G}(\lambda), \bar{B}(\lambda)$ of the spectral tri-stimulus values for each observer were obtained by repeating the color matching by MA five times at every wavelength.

(c) One of the 27 kinds of the mixtures composed of the three reference stimuli, whose intensities were $\bar{R}(\lambda)$ or $\bar{R}(\lambda) \pm \sigma_{R}(\lambda), \bar{G}(\lambda)$ or $\bar{G}(\lambda)$ $\pm \sigma_{G}(\lambda), \bar{B}(\lambda)$ or $\bar{B}(\lambda) \pm \sigma_{B}(\lambda)$, respectively, chosen in randomized order for the presentation to the lower half of the matching field. Chromaticity points of the 27 kinds of the mixture were, for example, as shown in Fig. 1 for a test spectrum of $500 \mathrm{~nm}$ in wavelength.

(d) After the preadaptation by the white light for 20 seconds, one of the 27 matching pairs with

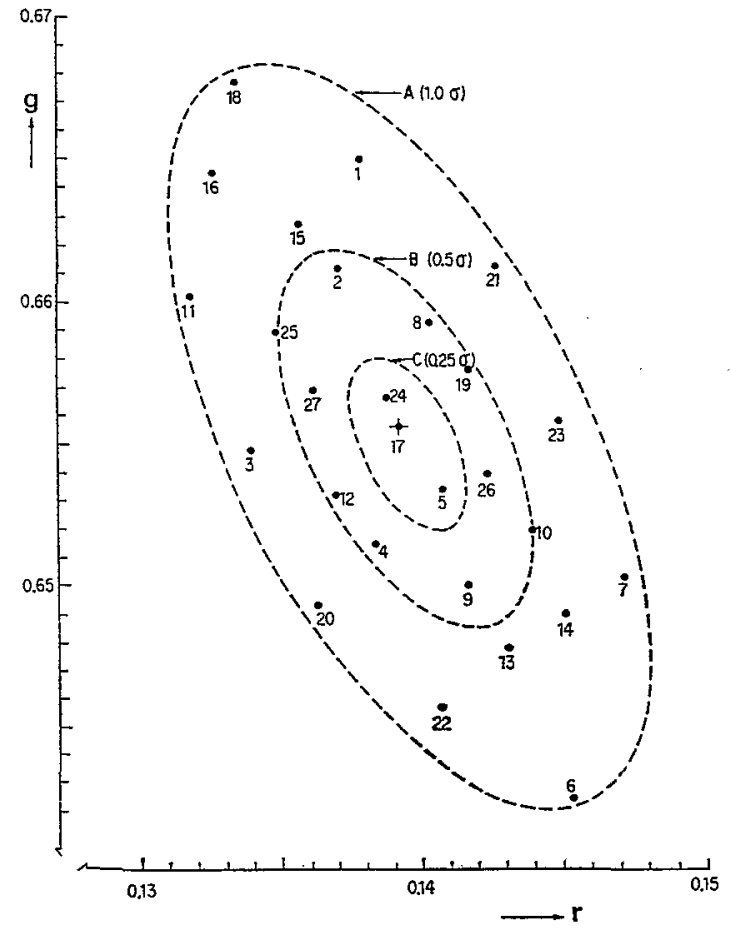

Fig. 1 Chromaticity points of the 27 kinds of the test color stimuli $(\lambda=500 \mathrm{~nm})$ with constant color differences. Numbers near the points represent the order according to which the test color stimuli were presented to the matching field for the color matching experiments by the method of constant stimuli, and the point of the number 17 represents the chromaticity coordinates corresponding to the mean tri-stimulus values measured by the method of adjustment.

the constant color differences was presented to the observer for 5 seconds.

(e) The degree of color matching for each of the matching pairs was evaluated by the categorical judgement.

(f) After the result of judgement was recorded, the experiments were repeated, returning to the previous step (c).

\subsection{Measurements and data processing}

Intensity $I(\lambda)$ of the test spectral stimulus $F(\lambda)$ of the wavelength $\lambda(\mathrm{nm})$ at the color match, and also the maximum intensities $I_{R}, I_{G}, I_{B}$ of the three instrumental reference stimuli at the origins of the respective wedges were measured in terms of the relative irradiance by a radiometric system with a phototube, whose relative spectral sensitivity was determined by calibration against a thermopile. The mean maximum intensities $L_{R}, L_{G}, L_{B}$ in terms of the retinal illuminance of the three instrumental referencestimuli. were also determined by the flicker photometry with three observers referring to a white light stimulus. The reference stimulus was 
reflected from an uniform diffuser illuminated by a standard lamp, whose luminous intensity had been evaluated.

The spectral tri-stimulus values $R(\lambda), G(\lambda)$, $B(\lambda)$, that is, the intensities of the instrumental reference stimuli required for the color match of the equi-energy spectrum, were evaluated by using the overall transmittances $\tau_{R}(\lambda), \tau_{B}(\lambda), \tau_{G}(\lambda)$ of the wedges and filters inserted at the color match, corresponding to the three reference stimuli, as follows :

$$
\begin{aligned}
& R(\lambda)=\tau_{R}(\lambda) \cdot I_{R} / I(\lambda), \\
& G(\lambda)=\tau_{G}(\lambda) \cdot I_{G} / I(\lambda), \\
& B(\lambda)=\tau_{B}(\lambda) \cdot I_{B} / I(\lambda) .
\end{aligned}
$$

Normalizing the respective spectral tri-stimulus values to make them equal to unity at the wavelengths of the corresponding reference stimuli, the color matching functions $\bar{r}(\lambda), \bar{g}(\lambda), \bar{b}(\lambda)$ for the equi-energy spectrum were obtained as follows:

$$
\begin{aligned}
& \bar{r}(\lambda)=R(\lambda) / R(670), \\
& \bar{g}(\lambda)=G(\lambda) / G(530), \\
& \bar{b}(\lambda)=B(\lambda) / B(460) .
\end{aligned}
$$

\section{Results and discussion}

\subsection{Additivity of color equations}

Using the six test spectral stimuli of the wavelengths $470,485,500,540,570$ and $600 \mathrm{~nm}$, it was examined whether or not the chromaticity coordinates of the color mixture composed of the test spectral stimulus $F(\lambda)$ of the wavelength $\lambda(\mathrm{nm})$ and the desaturating reference stimulus $D(\lambda)$ corresponding to $F(\lambda)$ were located at such points in

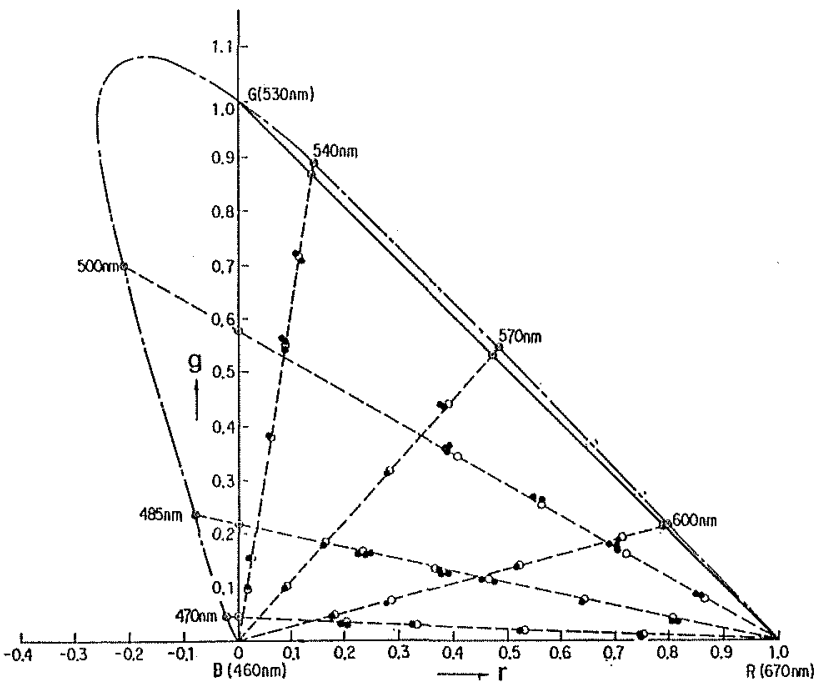

Fig. 2 Chromaticities of the color mixtures presented to the $2^{\circ}$ color matching field. They are composed of the test spectral stimulus and the desaturating reference stimulus in various rations. Dots represent the measured values of the chromaticity coordinates of the mixtures and open circles represent the chromaticity coordinates of the mixtures derived on the assumption that additivity holds.

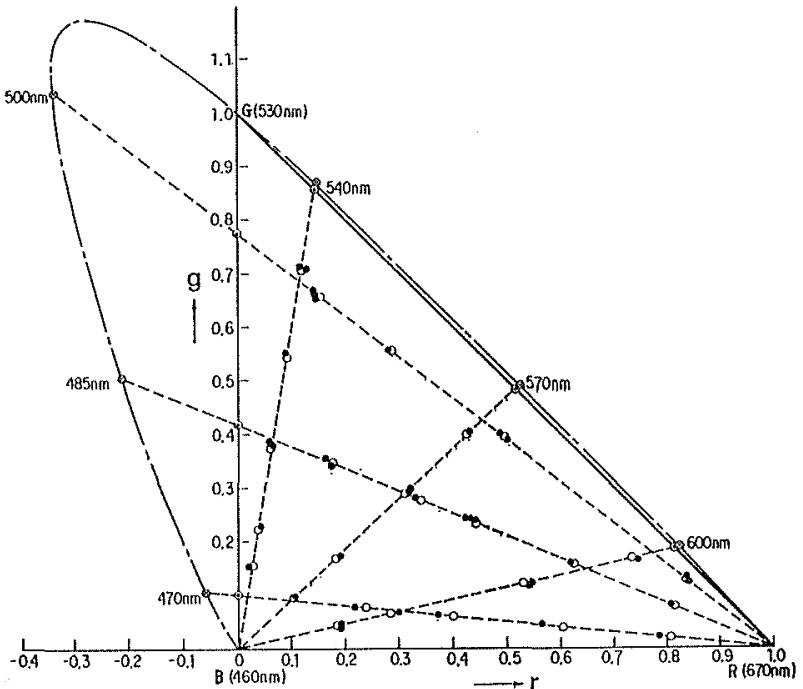

Fig. 3 Chromaticities of the color mixtures presented to the $10^{\circ}$ color matching field. They are composed of the test spectral strimulus and the desaturating reference stimulus in various ratios. Dots represent the measured values of the chromaticity coordinates of the mixtures and open circles represent the chromaticity coordinates of the mixtures derived on the assumption that additivity holds

the chromaticity diagram as the additivity law held.

Fig. 2 and Fig. 3 show the results of an observer (M.F.) for the $2^{\circ}$ and $10^{\circ}$ fields, respectively. In the figures, dots represent the measured values of the chromaticity coordinates of the color mixture in the color matching field, and open circles represent the estimated values derived on the assumtion of additivity. All the measured values laid on the straight lines joining the chromaticity coordinates of $F(\lambda)$ and $D(\lambda)$ in the chromaticity diagram.

Some of the previous researchers described that the additivity law held good in one condition ${ }^{8) 9}$, and others described that it did not so in other condition ${ }^{10) 11)}$. All the results of four observers in the present preliminary experiment showed that the additivity law held good for the $10^{\circ}$ field as well as the $2^{\circ}$ field, although the results of one observer were different a little from those of the others.

\subsection{Effect of chromatic adaptation}

Under the four kinds of the chromatic adaptation lights of red, green, blue and white colors, the degree of the color match in the four kinds of metameric pairs corresponding to the four test spectral stimuli of the wavelengths 500, 550,600 and $650(\mathrm{~nm})$ was evaluated by the categorical judgement. Results of the variance analysis of the evaluated values showed that the color match of one metameric pair broke down as the color of the chromatic adaptation light changed.

Crawford (1965) reported that the color matching functions were affected by the chromaticity of the matching field at the color match $^{12)}$, and 
Wyszecki (1978) also reported that they were dependent on the field intensity at moderate to high levels of retinal illuminance ${ }^{13)}$.

These results suggested that the color matching functions measured by the method of adjustment might have different values depending on the chromatic adaptation state to the color stimuli themselves of the matching field.

\subsection{Comparison of color matching functions}

Table 1 and Table 2 show the coefficients of deviation in percentages, that is, the ratios of the standard deviations among the values measured by the individual observer to the individual mean values of the two sets of the color matching functions obtained by MCS and MA in the columns 2, 3,4 and the columns 5, 6, 7, respectively, for an observer ( $Y$. Sh.). Comparing these two tables, it is seen that the deviations by the method of constant stimuli are less than those by the method of adjustment by about one tenth. And also, the relative deviations in percentages, that is, the ratios of the differences between the indivisual mean values by the method of adjustment and those by the method of constant stimuli to the latter, are tabulated in Table 2 for the same observer (Y. Sh.).

Table 1 Coefficients of deviation (\%) among the measured values of the indivisual color matching functions obtained by the method of constant stimuli and the method of adjustment for an observer (Y.Sh.).

\begin{tabular}{|c|c|c|c|c|c|c|c|c|}
\hline \multirow{2}{*}{$\begin{array}{c}\lambda \\
(\mathrm{nm})\end{array}$} & \multicolumn{3}{|c|}{ MCS } & \multicolumn{5}{|c|}{$\mathrm{MA}$} \\
\hline & $c_{r}(\lambda)$ & $c_{g}^{-}(\lambda)$ & $c_{b}(\lambda)$ & $c_{\bar{r}}(\lambda)$ & $c_{\bar{g}}(\lambda)$ & & $c_{b}(\lambda)$ & \\
\hline 395 & $0.00 \mathrm{E} \quad 0$ & $0.00 \mathrm{E} \quad 0$ & $0.00 \mathrm{E} \quad 0$ & $7.40 \mathrm{E}$ & $01.03 \mathrm{E}$ & 1 & $1.14 \mathrm{E}$ & 1 \\
\hline $\begin{array}{l}400 \\
410 \\
420 \\
430 \\
440 \\
450 \\
460 \\
470 \\
480 \\
490\end{array}$ & $\begin{array}{l}8.63 \mathrm{E}-1 \\
3.60 \mathrm{E}-0 \\
9.94 \mathrm{E}-1 \\
7.92 \mathrm{E}-1 \\
5.14 \mathrm{E}-1 \\
6.89 \mathrm{E}-1 \\
0.00 \mathrm{E}-0 \\
5.53 \mathrm{E}-1 \\
3.40 \mathrm{E}-1 \\
1.81 \mathrm{E}-1\end{array}$ & 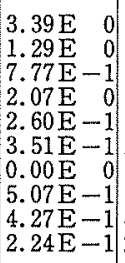 & $\begin{array}{l}7.64 \mathrm{E}-1 \\
4.06 \mathrm{E}-1 \\
4.74 \mathrm{E}-1 \\
5.61 \mathrm{E}-1 \\
5.53 \mathrm{E}-1 \\
2.58 \mathrm{E}-1 \\
5.70 \mathrm{E}-1 \\
5.47 \mathrm{E}-1 \\
4.19 \mathrm{E}-1 \\
2.74 \mathrm{E}-1\end{array}$ & $\begin{array}{l}6.19 \mathrm{E} \\
5.15 \mathrm{E} \\
7.38 \mathrm{E} \\
4.14 \mathrm{E} \\
5.04 \mathrm{E} \\
7.26 \mathrm{E} \\
0.00 \mathrm{E} \\
1.05 \mathrm{E} \\
2.28 \mathrm{E} \\
7.27 \mathrm{E}-1\end{array}$ & \begin{tabular}{l|l}
0 & $1.23 \mathrm{E}$ \\
0 & $9.16 \mathrm{E}$ \\
0 & $5.67 \mathrm{E}$ \\
0 & $3.78 \mathrm{E}$ \\
0 & $2.49 \mathrm{E}$ \\
0 & $5.85 \mathrm{E}$ \\
0 & $0.00 \mathrm{E}$ \\
1 & $2.99 \mathrm{E}$ \\
0 & $3.61 \mathrm{E}$ \\
1 & $2.01 \mathrm{E}$
\end{tabular} & & $\begin{array}{l}3.21 \mathrm{E} \\
2.63 \mathrm{E} \\
8.06 \mathrm{E} \\
3.98 \mathrm{E} \\
2.33 \mathrm{E} \\
2.41 \mathrm{E} \\
8.68 \mathrm{E}- \\
5.91 \mathrm{E} \\
2.79 \mathrm{E} \\
2.23 \mathrm{E}\end{array}$ & $\begin{array}{l}0 \\
0 \\
0 \\
0 \\
0 \\
0 \\
-1 \\
0 \\
0 \\
0\end{array}$ \\
\hline $\begin{array}{l}500 \\
510 \\
520 \\
530 \\
540 \\
550 \\
560 \\
570 \\
580 \\
590\end{array}$ & $\begin{array}{l}1.95 \mathrm{E}-1 \\
2.95 \mathrm{E}-1 \\
1.03 \mathrm{E} \quad 0 \\
0.00 \mathrm{E} \quad 0 \\
6.77 \mathrm{E}-1 \\
2.88 \mathrm{E}-1 \\
1.96 \mathrm{E}-1 \\
2.58 \mathrm{E}-1 \\
3.46 \mathrm{E}-1 \\
2.52 \mathrm{E}-1\end{array}$ & $\begin{array}{l}5.49 \mathrm{E}-1 \\
2.09 \mathrm{E}-1 \\
1.36 \mathrm{E}-1 \\
3.25 \mathrm{E}-1 \\
1.76 \mathrm{E}-1 \\
1.91 \mathrm{E}-1 \\
1.92 \mathrm{E}-1 \\
1.91 \mathrm{E}-1 \\
2.36 \mathrm{E}-1 \\
1.64 \mathrm{E}-1\end{array}$ & $\begin{array}{rr}2.58 \mathrm{E}-1 \\
2.77 \mathrm{E}-1 \\
4.21 \mathrm{E}-1 \\
0.00 \mathrm{E} & 0 \\
2.59 \mathrm{E} & 0 \\
2.27 \mathrm{E} & 0 \\
1.26 \mathrm{E} & 0 \\
8.63 \mathrm{E} & -1 \\
2.21 \mathrm{E} & 0 \\
7.44 \mathrm{E}-1\end{array}$ & 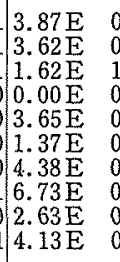 & \begin{tabular}{l|l}
0 & $1.89 \mathrm{E}$ \\
0 & $9.02 \mathrm{E}-$ \\
1 & $1.14 \mathrm{E}$ \\
0 & $2.17 \mathrm{E}$ \\
0 & $9.42 \mathrm{E}-$ \\
0 & $1.74 \mathrm{E}$ \\
0 & $1.43 \mathrm{E}$ \\
0 & $7.66 \mathrm{E}-$ \\
0 & $1.28 \mathrm{E}$ \\
0 & $3.77 \mathrm{E}$
\end{tabular} & 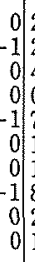 & $\begin{array}{l}2.75 \mathrm{E} \\
2.06 \mathrm{E} \\
4.71 \mathrm{E} \\
0.00 \mathrm{E} \\
7.26 \mathrm{E} \\
1.47 \mathrm{E} \\
1.32 \mathrm{E} \\
8.47 \mathrm{E} \\
2.45 \mathrm{E} \\
1.01 \mathrm{E}\end{array}$ & $\begin{array}{l}0 \\
0 \\
0 \\
0 \\
1 \\
1 \\
1 \\
0 \\
1 \\
1\end{array}$ \\
\hline $\begin{array}{l}600 \\
610 \\
620 \\
630 \\
640 \\
650 \\
660 \\
670 \\
680 \\
690\end{array}$ & $\begin{array}{l}2.83 \mathrm{E}-1 \\
2.28 \mathrm{E}-1 \\
2.34 \mathrm{E}-1 \\
2.84 \mathrm{E}-1 \\
3.21 \mathrm{E}-1 \\
2.69 \mathrm{E}-1 \\
3.39 \mathrm{E}-1 \\
2.37 \mathrm{E}-1 \\
1.84 \mathrm{E}-1 \\
5.20 \mathrm{E}-1\end{array}$ & 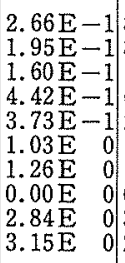 & $\mid \begin{array}{rr}8.34 \mathrm{E}-1 \\
8.23 \mathrm{E}-1 \\
1.03 \mathrm{E} & 0 \\
6.10 \mathrm{E}-1 \\
2.64 \mathrm{E} & 0 \\
6.99 \mathrm{E}-1 \\
1.85 \mathrm{E} & 0 \\
0.00 \mathrm{E} & 0 \\
3.53 \mathrm{E} & 0 \\
2.42 \mathrm{E} & 0\end{array}$ & 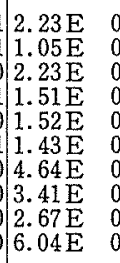 & \begin{tabular}{l|l}
0 & $2.47 \mathrm{E}$ \\
0 & $1.61 \mathrm{E}$ \\
0 & $3.31 \mathrm{E}$ \\
0 & $1.84 \mathrm{E}$ \\
0 & $2.35 \mathrm{E}$ \\
0 & $3.27 \mathrm{E}$ \\
0 & $1.24 \mathrm{E}$ \\
0 & $0.00 \mathrm{E}$ \\
0 & $1.92 \mathrm{E}$ \\
0 & $1.82 \mathrm{E}$
\end{tabular} & $\begin{array}{l}0 \\
0 \\
0 \\
0 \\
0 \\
0 \\
1 \\
0 \\
1 \\
1\end{array}$ & $\begin{array}{l}8.21 \mathrm{E} \\
1.54 \mathrm{E} \\
1.26 \mathrm{E} \\
1.09 \mathrm{E} \\
1.38 \mathrm{E} \\
1.29 \mathrm{E} \\
4.34 \mathrm{E} \\
0.00 \mathrm{E} \\
2.92 \mathrm{E} \\
1.43 \mathrm{E}\end{array}$ & $\begin{array}{l}0 \\
1 \\
1 \\
1 \\
1 \\
1 \\
1 \\
0 \\
0 \\
1\end{array}$ \\
\hline $\begin{array}{l}700 \\
720 \\
740\end{array}$ & $\begin{array}{l}\text { 1. } 46 \mathrm{E}-1 \\
\text { 3. } 48 \mathrm{E}-1 \\
1.38 \mathrm{E}-1\end{array}$ & 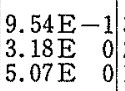 & $\begin{array}{ll}3.95 \mathrm{E} & 0 \\
2.46 \mathrm{E} & 0 \\
1.91 \mathrm{E} & 0\end{array}$ & $\begin{array}{ll}3.11 \mathrm{E} & 0 \\
2.85 \mathrm{E} & 0 \\
3.05 \mathrm{E} & 0\end{array}$ & \begin{tabular}{l|l}
0 & $7.14 \mathrm{E}$ \\
0 & $1.17 \mathrm{E}$ \\
0 & $1.93 \mathrm{E}$
\end{tabular} & & $\begin{array}{l}3.78 \mathrm{E} \\
1.38 \mathrm{E} \\
2.19 \mathrm{E}\end{array}$ & $\begin{array}{l}1 \\
1 \\
1\end{array}$ \\
\hline
\end{tabular}

Table 2 Relative deviations (\%) of the indivisual color matching functions by the method of adjustment from those by the method of constant stimuli for the observer ( $Y$. Sh.).

\begin{tabular}{|c|c|c|c|c|}
\hline$\lambda(\mathrm{nm})$ & $\Delta \bar{r}(\lambda)$ & $\Delta \ddot{g}(\lambda)$ & & $\Delta \bar{b}(\lambda)$ \\
\hline 395 & $-5.41 \mathrm{E} \quad 0$ & $8.24 \mathrm{E}$ & 0 & $-1.29 \mathrm{E} \quad 1$ \\
\hline $\begin{array}{l}400 \\
410 \\
420 \\
430 \\
440 \\
450 \\
460 \\
470 \\
480 \\
490\end{array}$ & $\begin{array}{rr}8.67 \mathrm{E} & 0 \\
3.76 \mathrm{E} & 0 \\
-5.81 \mathrm{E} & 0 \\
-2.38 \mathrm{E} & 0 \\
-3.75 \mathrm{E} & 0 \\
-4.32 \mathrm{E} & 0 \\
0.00 \mathrm{E} & 0 \\
-1.15 \mathrm{E} & 1 \\
-4.89 \mathrm{E}-1 \\
-2.03 \mathrm{E}-1\end{array}$ & $\begin{array}{r}1.93 \mathrm{E} \\
2.96 \mathrm{E} \\
-7.55 \mathrm{E} \\
-2.74 \mathrm{E} \\
-7.01 \mathrm{E}-1 \\
-6.74 \mathrm{E} \\
0.00 \mathrm{E} \\
8.25 \mathrm{E}-1 \\
3.60 \mathrm{E} \\
2.17 \mathrm{E}\end{array}$ & $\begin{array}{r}1 \\
0 \\
0 \\
0 \\
-1 \\
0 \\
0 \\
-1 \\
0 \\
0\end{array}$ & $\begin{array}{rr}7.83 \mathrm{E} & 0 \\
-1.32 \mathrm{E} & 1 \\
-1.14 \mathrm{E} & 1 \\
-1.32 \mathrm{E} & 1 \\
-9.72 \mathrm{E} & 0 \\
-1.21 \mathrm{E} & 1 \\
0.00 \mathrm{E} & 0 \\
1.15 \mathrm{E} & 1 \\
1.39 \mathrm{E}-1 \\
3.01 \mathrm{E} & 0\end{array}$ \\
\hline $\begin{array}{l}500 \\
510 \\
520 \\
530 \\
540 \\
550 \\
560 \\
570 \\
580 \\
590\end{array}$ & $\begin{array}{rr}4.38 \mathrm{E} & 0 \\
-4.63 \mathrm{E} & 0 \\
-1.57 \mathrm{E} & 1 \\
0.00 \mathrm{E} & 0 \\
1.89 \mathrm{E} & 0 \\
-7.25 \mathrm{E} & 0 \\
-1.83 \mathrm{E} & 0 \\
-6.75 \mathrm{E} & 0 \\
-3.36 \mathrm{E} & 0 \\
-9.37 \mathrm{E} & -1\end{array}$ & $\begin{array}{r}6.03 \mathrm{E} \\
-2.51 \mathrm{E} \\
1.27 \mathrm{E} \\
0.00 \mathrm{E} \\
-2.95 \mathrm{E}-1 \\
3.22 \mathrm{E}- \\
-1.15 \mathrm{E} \\
-3.69 \mathrm{E} \\
-5.06 \mathrm{E} \\
-4.96 \mathrm{E}\end{array}$ & $\begin{array}{r}0 \\
0 \\
0 \\
0 \\
-1 \\
-2 \\
0 \\
0 \\
0 \\
0\end{array}$ & $\begin{array}{rl}3.59 \mathrm{E}-1 \\
-1.14 \mathrm{E} & 0 \\
-5.17 \mathrm{E} & 0 \\
0.00 \mathrm{E} & 0 \\
4.01 \mathrm{E}-2 \\
4.08 \mathrm{E} & 0 \\
1.45 \mathrm{E} & 1 \\
4.90 \mathrm{E} & 0 \\
4.40 \mathrm{E} & 0 \\
4.01 \mathrm{E} & 0\end{array}$ \\
\hline $\begin{array}{l}600 \\
610 \\
620 \\
630 \\
640 \\
650 \\
660 \\
670 \\
680 \\
690\end{array}$ & $\begin{array}{rr}-2.69 \mathrm{E} & 0 \\
-3.24 \mathrm{E} & -1 \\
-3.57 \mathrm{E} & 0 \\
-7.18 \mathrm{E} & 0 \\
-7.49 \mathrm{E} & 0 \\
-2.81 \mathrm{E} & 0 \\
-2.42 \mathrm{E} & 0 \\
0.00 \mathrm{E} & 0 \\
-1.06 \mathrm{E} & 0 \\
-8.83 \mathrm{E} & -1\end{array}$ & $\begin{array}{r}-5.94 \mathrm{E} \\
-3.39 \mathrm{E} \\
-1.18 \mathrm{E} \\
-1.04 \mathrm{E} \\
-1.08 \mathrm{E} \\
-1.02 \mathrm{E} \\
4.11 \mathrm{E} \\
0.00 \mathrm{E} \\
3.25 \mathrm{E} \\
-2.76 \mathrm{E}\end{array}$ & $\begin{array}{l}0 \\
0 \\
1 \\
1 \\
1 \\
1 \\
0 \\
0 \\
0 \\
0\end{array}$ & $\begin{array}{rr}5.13 \mathrm{E} & 0 \\
5.69 \mathrm{E} & 0 \\
-5.98 \mathrm{E} & 0 \\
4.90 \mathrm{E} & 1 \\
-4.61 \mathrm{E} & 0 \\
-3.71 \mathrm{E} & 0 \\
7.86 \mathrm{E}-1 \\
0.00 \mathrm{E} & 0 \\
-3.74 \mathrm{E} & 0 \\
-5.37 \mathrm{E} & 0\end{array}$ \\
\hline $\begin{array}{l}700 \\
720 \\
740\end{array}$ & $\begin{array}{rl}-4.28 \mathrm{E} & 0 \\
-9.92 \mathrm{E} & 0 \\
3.61 \mathrm{E} & 0\end{array}$ & $\begin{array}{l}1.07 \mathrm{E} \\
9.05 \mathrm{E} \\
0.00 \mathrm{E}\end{array}$ & $\begin{array}{l}3 \\
1 \\
0\end{array}$ & $\begin{array}{rl}1.45 \mathrm{E} & 2 \\
-5.74 \mathrm{E} & 0 \\
5.16 \mathrm{E} & 1\end{array}$ \\
\hline
\end{tabular}

Since these relative deviations are larger than the coefficients of deviation in the individual color matching functions by the method of constant stimuli, it can be said that in respect to the individual observer, the color matching functions obtained by one method were obiously different from those by the other method.

There were, however, no significant differences between the mean values taken over all the observers by one method and those by the other method, since the differences among the ten sets of the indivisual color matching functions for ten observers by either of two methods were larger than the differences between the two sets of the color matching functions for each observer obtained by the two methods.

The over-all mean values of the two sets of the ETL $10^{\circ}$ color matching functions by MCS and MA are tabulated in Table 3 and Table 4, respectively, with the coefficients of deviations in percentages, that is, the ratios of the standard deviations among the individual mean values to the over-all mean values, and the mean values and standard deviations of the field intensities in trolands at the color match.

The relative deviations in percentages between the over-all mean color matching functions by MA and those by MCS are tabulated in Table 5.

And also, the relative deviations in percentages between the ETL mean data by MCS and the CIE 
Table 3 The ETL $10^{\circ}$ color matching functions determined by the method of constant stimuli. $\vec{r}(\lambda), \bar{g}(\lambda), \bar{b}(\lambda)$ : the over-all mean values obtained by averaging the indivisual mean color. matching functions of ten observers; $c_{\bar{r}}(\lambda), c_{\bar{y}}(\lambda), c_{\bar{b}}(\lambda)$ : the coefficients of deviation (\%) among the indivisual mean functions; $T(\lambda), \sigma_{T}(\lambda)$ : the mean value and the standard deviation of the field intensity (trolands) at the color match, respectively.

\begin{tabular}{|c|c|c|c|c|c|c|c|c|c|c|c|}
\hline$\lambda(\mathrm{nm})$ & $\bar{\gamma}(\lambda)$ & $\bar{g}(\lambda)$ & $\bar{b}(\lambda)$ & $c_{r}(\lambda)$ & & $c_{g}^{-}(\lambda)$ & & $c_{\bar{b}}(\lambda)$ & & $T(\lambda)(\operatorname{tr} d)$ & $\sigma_{T}(\lambda)(\operatorname{trd})$ \\
\hline 395 & 1. $400 \mathrm{E}-2$ & $-1.545 E-3$ & $1.919 \mathrm{E}-2$ & $6.90 \mathrm{E}$ & 1 & $4.74 \mathrm{E}$ & 1 & $6.28 \mathrm{E}$ & 1 & 2.00 & 1.98 \\
\hline $\begin{array}{l}400 \\
410 \\
420 \\
430 \\
440 \\
450 \\
460 \\
470 \\
480 \\
490\end{array}$ & $\begin{array}{r}4.068 \mathrm{E}-2 \\
1.470 \mathrm{E}-1 \\
3.440 \mathrm{E}-1 \\
5.192 \mathrm{E}-1 \\
5.923 \mathrm{E}-1 \\
3.623 \mathrm{E}-1 \\
0.000 \mathrm{E} \quad 0 \\
-6.062 \mathrm{E}-1 \\
-1.264 \mathrm{E} \quad 0 \\
-1.718 \mathrm{E} \quad 0\end{array}$ & $\begin{array}{r}-5.628 \mathrm{E}-3 \\
-2.266 \mathrm{E}-2 \\
-5.326 \mathrm{E}-2 \\
-8.173 \mathrm{E}-2 \\
-1.054 \mathrm{E}-1 \\
-6.087 \mathrm{E}-2 \\
0.000 \mathrm{E}-0 \\
1.134 \mathrm{E}-1 \\
2.498 \mathrm{E}-1 \\
3.963 \mathrm{E}-1\end{array}$ & $\begin{array}{l}5.216 \mathrm{E}-2 \\
2.046 \mathrm{E}-1 \\
4.754 \mathrm{E}-1 \\
7.976 \mathrm{E}-1 \\
1.202 \mathrm{E} \quad 0 \\
1.146 \mathrm{E} \quad 0 \\
1.000 \mathrm{E} \quad 0 \\
7.381 \mathrm{E}-1 \\
4.369 \mathrm{E}-1 \\
2.376 \mathrm{E}-1\end{array}$ & $\begin{array}{l}5.61 \mathrm{E} \\
3.24 \mathrm{E} \\
2.16 \mathrm{E} \\
2.19 \mathrm{E} \\
1.95 \mathrm{E} \\
2.37 \mathrm{E} \\
0.00 \mathrm{E} \\
1.77 \mathrm{E} \\
1.46 \mathrm{E} \\
1.31 \mathrm{E}\end{array}$ & $\begin{array}{l}1 \\
1 \\
1 \\
1 \\
1 \\
1 \\
0 \\
1 \\
1 \\
1\end{array}$ & $\begin{array}{l}4.98 \mathrm{E} \\
3.20 \mathrm{E} \\
2.10 \mathrm{E} \\
1.89 \mathrm{E} \\
1.69 \mathrm{E} \\
2.31 \mathrm{E} \\
0.00 \mathrm{E} \\
1.12 \mathrm{E} \\
7.13 \mathrm{E} \\
7.66 \mathrm{E}\end{array}$ & $\begin{array}{l}1 \\
1 \\
1 \\
1 \\
1 \\
1 \\
0 \\
1 \\
0 \\
0\end{array}$ & $\begin{array}{l}4.73 \mathrm{E} \\
2.71 \mathrm{E} \\
1.42 \mathrm{E} \\
1.37 \mathrm{E} \\
1.20 \mathrm{E} \\
6.92 \mathrm{E} \\
0.00 \mathrm{E} \\
6.17 \mathrm{E} \\
9.10 \mathrm{E} \\
6.96 \mathrm{E}\end{array}$ & $\begin{array}{l}1 \\
1 \\
1 \\
1 \\
1 \\
0 \\
0 \\
0 \\
0 \\
0\end{array}$ & $\begin{array}{r}13.39 \\
99.59 \\
161.49 \\
165.87 \\
190.00 \\
194.85 \\
178.05 \\
232.71 \\
220.29 \\
247.89\end{array}$ & $\begin{array}{r}5.45 \\
5.55 \\
14.82 \\
13.28 \\
10.91 \\
4.15 \\
3.08 \\
5.13 \\
5.69 \\
6.76\end{array}$ \\
\hline $\begin{array}{l}500 \\
510 \\
520 \\
530 \\
540 \\
550 \\
560 \\
570 \\
580 \\
590\end{array}$ & $\begin{array}{rl}-1.844 \mathrm{E} & 0 \\
-1.820 \mathrm{E} & 0 \\
-1.248 \mathrm{E} & 0 \\
0.000 \mathrm{E} & 0 \\
1.349 \mathrm{E} & 0 \\
3.066 \mathrm{E} & 0 \\
5.252 \mathrm{E} & 0 \\
7.257 \mathrm{E} & 0 \\
9.401 \mathrm{E} & 0 \\
1.095 \mathrm{E} & 1\end{array}$ & $\begin{array}{l}5.551 \mathrm{E}-1 \\
7.327 \mathrm{E}-1 \\
8.856 \mathrm{E}-1 \\
1.000 \mathrm{E} \quad 0 \\
1.053 \mathrm{E} \quad 0 \\
1.028 \mathrm{E} \quad 0 \\
9.541 \mathrm{E}-1 \\
8.293 \mathrm{E}-1 \\
6.563 \mathrm{E}-1 \\
4.701 \mathrm{E}-1\end{array}$ & $\begin{array}{r}1.247 \mathrm{E}-1 \\
5.922 \mathrm{E}-2 \\
2.685 \mathrm{E}-2 \\
0.000 \mathrm{E}-0 \\
-6.193 \mathrm{E}-3 \\
-1.124 \mathrm{E}-2 \\
-1.341 \mathrm{E}-2 \\
-1.441 \mathrm{E}-2 \\
-1.492 \mathrm{E}-2 \\
-1.513 \mathrm{E}-2\end{array}$ & $\begin{array}{l}7.55 \mathrm{E} \\
5.81 \mathrm{E} \\
1.56 \mathrm{E} \\
0.00 \mathrm{E} \\
9.86 \mathrm{E} \\
8.51 \mathrm{E} \\
6.66 \mathrm{E} \\
4.39 \mathrm{E} \\
4.51 \mathrm{E} \\
4.40 \mathrm{E}\end{array}$ & $\begin{array}{l}0 \\
0 \\
1 \\
0 \\
0 \\
0 \\
0 \\
0 \\
0 \\
0\end{array}$ & $\begin{array}{l}5.62 \mathrm{E} \\
5.34 \mathrm{E} \\
2.66 \mathrm{E} \\
0.00 \mathrm{E} \\
1.63 \mathrm{E} \\
2.48 \mathrm{E} \\
2.42 \mathrm{E} \\
2.87 \mathrm{E} \\
4.75 \mathrm{E} \\
5.64 \mathrm{E}\end{array}$ & $\begin{array}{l}0 \\
0 \\
0 \\
0 \\
0 \\
0 \\
0 \\
0 \\
0 \\
0\end{array}$ & $\begin{array}{l}5.74 \mathrm{E} \\
9.65 \mathrm{E} \\
1.65 \mathrm{E} \\
0.00 \mathrm{E} \\
1.14 \mathrm{E} \\
1.29 \mathrm{E} \\
1.43 \mathrm{E} \\
1.37 \mathrm{E} \\
1.34 \mathrm{E} \\
1.27 \mathrm{E}\end{array}$ & $\begin{array}{l}0 \\
0 \\
1 \\
0 \\
1 \\
1 \\
1 \\
1 \\
1 \\
1\end{array}$ & $\begin{array}{l}253.13 \\
262.91 \\
252.91 \\
228.75 \\
202.75 \\
216.17 \\
220.16 \\
214.11 \\
215.54 \\
205.06\end{array}$ & $\begin{array}{l}5.86 \\
5.62 \\
2.92 \\
3.48 \\
1.72 \\
2.39 \\
3.12 \\
2.64 \\
2.94 \\
3.95\end{array}$ \\
\hline $\begin{array}{l}600 \\
610 \\
620 \\
630 \\
640 \\
650 \\
660 \\
670 \\
680 \\
690\end{array}$ & $\begin{array}{ll}1.161 \mathrm{E} & 1 \\
1.100 \mathrm{E} & 1 \\
9.507 \mathrm{E} & 0 \\
7.611 \mathrm{E} & 0 \\
5.229 \mathrm{E} & 0 \\
3.379 \mathrm{E} & 0 \\
1.914 \mathrm{E} & 0 \\
1.000 \mathrm{E} & 0 \\
5.311 \mathrm{E}-1 \\
2.645 \mathrm{E}-1\end{array}$ & $\begin{array}{r}2.969 \mathrm{E}-1 \\
1.739 \mathrm{E}-1 \\
9.307 \mathrm{E}-2 \\
3.992 \mathrm{E}-2 \\
1.514 \mathrm{E}-2 \\
5.535 \mathrm{E}-3 \\
1.220 \mathrm{E}-3 \\
0.000 \mathrm{E}-0 \\
-1.556 \mathrm{E}-4 \\
-9.426 \mathrm{E}-5\end{array}$ & $\begin{array}{r}-1.420 \mathrm{E}-2 \\
-1.114 \mathrm{E}-2 \\
-8.119 \mathrm{E}-3 \\
-3.980 \mathrm{E}-3 \\
-2.140 \mathrm{E}-3 \\
-8.775 \mathrm{E}-4 \\
-4.210 \mathrm{E}-4 \\
0.000 \mathrm{E} 0 \\
1.055 \mathrm{E}-4 \\
5.318 \mathrm{E}-5\end{array}$ & $\begin{array}{l}3.40 \mathrm{E} \\
1.98 \mathrm{E} \\
1.44 \mathrm{E} \\
5.69 \mathrm{E} \\
3.22 \mathrm{E} \\
5.69 \mathrm{E} \\
3.96 \mathrm{E} \\
0.00 \mathrm{E} \\
5.13 \mathrm{E} \\
9.31 \mathrm{E}\end{array}$ & $\begin{array}{l}0 \\
0 \\
0 \\
0 \\
0 \\
0 \\
0 \\
0 \\
0 \\
0\end{array}$ & $\begin{array}{l}8.14 \mathrm{E} \\
7.61 \mathrm{E} \\
8.62 \mathrm{E} \\
1.06 \mathrm{E} \\
1.27 \mathrm{E} \\
1.33 \mathrm{E} \\
2.77 \mathrm{E} \\
0.00 \mathrm{E} \\
4.72 \mathrm{E} \\
3.79 \mathrm{E}\end{array}$ & $\begin{array}{l}0 \\
0 \\
0 \\
1 \\
1 \\
1 \\
1 \\
0 \\
1 \\
1\end{array}$ & $\begin{array}{l}1.33 \mathrm{E} \\
1.63 \mathrm{E} \\
1.71 \mathrm{E} \\
2.76 \mathrm{E} \\
2.20 \mathrm{E} \\
4.11 \mathrm{E} \\
4.85 \mathrm{E} \\
0.00 \mathrm{E} \\
1.01 \mathrm{E} \\
5.50 \mathrm{E}\end{array}$ & $\begin{array}{l}1 \\
1 \\
1 \\
1 \\
1 \\
1 \\
1 \\
1 \\
0 \\
2 \\
1\end{array}$ & $\begin{array}{l}193.53 \\
183.49 \\
200.94 \\
200.47 \\
198.26 \\
212.34 \\
211.92 \\
201.73 \\
255.46 \\
209.86\end{array}$ & $\begin{array}{l}3.74 \\
2.64 \\
4.01 \\
4.48 \\
5.38 \\
4.23 \\
5.74 \\
3.89 \\
4.31 \\
7.73\end{array}$ \\
\hline $\begin{array}{l}700 \\
720 \\
740\end{array}$ & $\begin{array}{l}1.196 \mathrm{E}-1 \\
2.660 \mathrm{E}-2 \\
5.593 \mathrm{E}-3\end{array}$ & $\begin{array}{l}-5.754 \mathrm{E}-5 \\
-2.390 \mathrm{E}-5 \\
-2.083 \mathrm{E}-6\end{array}$ & $\begin{array}{l}1.059 \mathrm{E}-5 \\
3.433 \mathrm{E}-6 \\
8.594 \mathrm{E}-7\end{array}$ & $\begin{array}{l}4.77 \mathrm{E} \\
7.00 \mathrm{E} \\
1.02 \mathrm{E}\end{array}$ & $\begin{array}{l}0 \\
0 \\
1\end{array}$ & $\begin{array}{l}4.54 \mathrm{E} \\
1.43 \mathrm{E} \\
4.17 \mathrm{E}\end{array}$ & $\begin{array}{l}1 \\
2 \\
1\end{array}$ & $\begin{array}{l}1.12 \mathrm{E} \\
4.76 \mathrm{E} \\
5.15 \mathrm{E}\end{array}$ & $\begin{array}{l}2 \\
1 \\
1\end{array}$ & $\begin{array}{r}200.24 \\
164.03 \\
78.64\end{array}$ & $\begin{array}{r}5.08 \\
10.50 \\
7.19\end{array}$ \\
\hline
\end{tabular}

Table 4 The ETL $10^{\circ}$ color matching functions determined by the method of adjustment.

$\vec{r}(\lambda), \vec{g}(\lambda), \vec{b}(\lambda)$ : the over-all mean values obtained by averaging the indivisual mean color matching functions of ten observers;

$c_{\bar{r}}(\lambda), c_{\bar{y}}(\lambda), c_{\bar{b}}(\lambda)$ : the coefficients of deviation (\%) among the indivisual mean fuctions; $T(\lambda), \sigma_{T}(\lambda)$ : the mean value and the standard deviation of the field intensity (trolands) at the color match, respectively.

\begin{tabular}{|c|c|c|c|c|c|c|c|c|c|c|c|}
\hline$\lambda(\mathrm{nm})$ & $\bar{r}(\lambda)$ & $\bar{g}(\lambda)$ & $\bar{b}(\lambda)$ & $c_{\bar{r}}(\lambda)$ & & $c_{\overline{0}}(\lambda)$ & & $c_{\bar{b}}(\lambda)$ & & $T(\lambda)(\operatorname{trd})$ & $\sigma_{T}(\lambda)(\operatorname{trd})$ \\
\hline 395 & 1. $588 \mathrm{E}-2$ & $-1.795 E-3$ & $1.907 \mathrm{E}-2$ & $8.62 \mathrm{E}$ & 1 & $6.13 \mathrm{E}$ & 1 & $6.44 \mathrm{E}$ & 1 & 2.81 & 2.86 \\
\hline $\begin{array}{l}400 \\
410 \\
420 \\
430 \\
440 \\
450 \\
460 \\
470 \\
480 \\
490\end{array}$ & $\begin{array}{r}3.929 \mathrm{E}-2 \\
1.651 \mathrm{E}-1 \\
3.479 \mathrm{E}-1 \\
5.306 \mathrm{E}-1 \\
5.835 \mathrm{E}-1 \\
3.624 \mathrm{E}-1 \\
0.000 \mathrm{E} \quad 0 \\
-5.990 \mathrm{E}-1 \\
-1.245 \mathrm{E} \quad 0 \\
-1.695 \mathrm{E} \quad 0\end{array}$ & $\begin{array}{r}-5.813 \mathrm{E}-3 \\
-2.518 \mathrm{E}-2 \\
-5.331 \mathrm{E}-2 \\
-8.391 \mathrm{E}-2 \\
-1.052 \mathrm{E}-1 \\
-6.174 \mathrm{E}-2 \\
0.000 \mathrm{E}-0 \\
1.156 \mathrm{E}-1 \\
2.515 \mathrm{E}-1 \\
3.963 \mathrm{E}-1\end{array}$ & $\begin{array}{l}5.093 \mathrm{E}-2 \\
2.054 \mathrm{E}-1 \\
4.541 \mathrm{E}-1 \\
7.761 \mathrm{E}-1 \\
1.135 \mathrm{E} \quad 0 \\
1.099 \mathrm{E} \quad 0 \\
1.000 \mathrm{E} 0 \\
7.398 \mathrm{E}-1 \\
4.356 \mathrm{E}-1 \\
2.349 \mathrm{E}-1\end{array}$ & $\begin{array}{l}6.98 \mathrm{E} \\
3.81 \mathrm{E} \\
2.48 \mathrm{E} \\
1.90 \mathrm{E} \\
1.85 \mathrm{E} \\
2.12 \mathrm{E} \\
0.00 \mathrm{E} \\
1.61 \mathrm{E} \\
1.57 \mathrm{E} \\
1.37 \mathrm{E}\end{array}$ & $\begin{array}{l}1 \\
1 \\
1 \\
1 \\
1 \\
1 \\
0 \\
1 \\
1 \\
1\end{array}$ & $\begin{array}{l}6.08 \mathrm{E} \\
3.61 \mathrm{E} \\
2.25 \mathrm{E} \\
1.58 \mathrm{E} \\
1.76 \mathrm{E} \\
1.70 \mathrm{E} \\
0.00 \mathrm{E} \\
1.19 \mathrm{E} \\
8.81 \mathrm{E} \\
8.91 \mathrm{E}\end{array}$ & $\begin{array}{l}1 \\
1 \\
1 \\
1 \\
1 \\
1 \\
0 \\
1 \\
0 \\
0\end{array}$ & $\begin{array}{l}5.92 \mathrm{E} \\
3.00 \mathrm{E} \\
1.52 \mathrm{E} \\
1.25 \mathrm{E} \\
1.14 \mathrm{E} \\
3.27 \mathrm{E} \\
0.00 \mathrm{E} \\
4.99 \mathrm{E} \\
4.90 \mathrm{E} \\
8.28 \mathrm{E}\end{array}$ & $\begin{array}{l}1 \\
1 \\
1 \\
1 \\
1 \\
1 \\
0 \\
0 \\
0 \\
0 \\
0\end{array}$ & $\begin{array}{r}13.04 \\
95.83 \\
156.74 \\
160.51 \\
181.02 \\
187.45 \\
177.53 \\
234.86 \\
221.23 \\
247.54\end{array}$ & $\begin{array}{r}7.45 \\
6.27 \\
15.15 \\
12.81 \\
9.69 \\
3.71 \\
3.09 \\
5.75 \\
6.23 \\
7.73\end{array}$ \\
\hline $\begin{array}{l}500 \\
510 \\
520 \\
530 \\
540 \\
550 \\
560 \\
570 \\
580 \\
590\end{array}$ & $\begin{array}{rl}-1.818 \mathrm{E} & 0 \\
-1.801 \mathrm{E} & 0 \\
-1.226 \mathrm{E} & 0 \\
0.000 \mathrm{E} & 0 \\
1.298 \mathrm{E} & 0 \\
3.000 \mathrm{E} & 0 \\
5.135 \mathrm{E} & 0 \\
7.274 \mathrm{E} & 0 \\
9.267 \mathrm{E} & 0 \\
1.081 \mathrm{E} & 1\end{array}$ & $\begin{array}{l}5.654 \mathrm{E}-1 \\
7.399 \mathrm{E}-1 \\
8.942 \mathrm{E}-1 \\
1.000 \mathrm{E} \quad 0 \\
1.047 \mathrm{E} \quad 0 \\
1.018 \mathrm{E} \quad 0 \\
9.450 \mathrm{E}-1 \\
8.090 \mathrm{E}-1 \\
6.457 \mathrm{E}-1 \\
4.624 \mathrm{E}-1\end{array}$ & $\begin{array}{r}1.245 \mathrm{E}-1 \\
5.631 \mathrm{E}-2 \\
2.362 \mathrm{E}-2 \\
0.000 \mathrm{E}-0 \\
-7.279 \mathrm{E}-3 \\
-1.265 \mathrm{E}-2 \\
-1.470 \mathrm{E}-2 \\
-1.582 \mathrm{E}-2 \\
-1.631 \mathrm{E}-2 \\
-1.616 \mathrm{E}-2\end{array}$ & $\begin{array}{l}9.09 \mathrm{E} \\
7.09 \mathrm{E} \\
1.71 \mathrm{E} \\
0.00 \mathrm{E} \\
8.54 \mathrm{E} \\
9.52 \mathrm{E} \\
7.06 \mathrm{E} \\
6.43 \mathrm{E} \\
5.09 \mathrm{E} \\
3.63 \mathrm{E}\end{array}$ & $\begin{array}{l}0 \\
0 \\
1 \\
0 \\
0 \\
0 \\
0 \\
0 \\
0 \\
0\end{array}$ & $\begin{array}{l}6.58 \mathrm{E} \\
4.84 \mathrm{E} \\
2.88 \mathrm{E} \\
0.00 \mathrm{E} \\
1.09 \mathrm{E} \\
1.28 \mathrm{E} \\
2.02 \mathrm{E} \\
2.75 \mathrm{E} \\
4.68 \mathrm{E} \\
6.09 \mathrm{E}\end{array}$ & $\begin{array}{l}0 \\
0 \\
0 \\
0 \\
0 \\
0 \\
0 \\
0 \\
0 \\
0\end{array}$ & $\begin{array}{l}9.21 \mathrm{E} \\
8.98 \mathrm{E} \\
1.45 \mathrm{E} \\
0.00 \mathrm{E} \\
1.26 \mathrm{E} \\
1.71 \mathrm{E} \\
1.38 \mathrm{E} \\
1.30 \mathrm{E} \\
1.29 \mathrm{E} \\
1.35 \mathrm{E}\end{array}$ & $\begin{array}{l}0 \\
0 \\
1 \\
0 \\
1 \\
1 \\
1 \\
1 \\
1 \\
1\end{array}$ & $\begin{array}{l}257.31 \\
264.96 \\
254.95 \\
228.66 \\
201.26 \\
213.97 \\
217.93 \\
210.74 \\
212.94 \\
203.05\end{array}$ & $\begin{array}{l}6.32 \\
5.13 \\
3.74 \\
4.04 \\
2.13 \\
2.52 \\
3.53 \\
2.97 \\
3.26 \\
4.35\end{array}$ \\
\hline $\begin{array}{l}600 \\
610 \\
620 \\
630 \\
640 \\
650 \\
660 \\
670 \\
680 \\
690\end{array}$ & $\begin{array}{ll}1.146 \mathrm{E} & 1 \\
1.088 \mathrm{E} & 1 \\
9.444 \mathrm{E} & 0 \\
7.456 \mathrm{E} & 0 \\
5.166 \mathrm{E} & 0 \\
3.318 \mathrm{E} & 0 \\
1.897 \mathrm{E} & 0 \\
1.000 \mathrm{E} & 0 \\
5.235 \mathrm{E}-1 \\
2.553 \mathrm{E}-1\end{array}$ & $\begin{array}{r}2.940 \mathrm{E}-1 \\
1.694 \mathrm{E}-1 \\
9.017 \mathrm{E}-2 \\
3.906 \mathrm{E}-2 \\
1.543 \mathrm{E}-2 \\
5.318 \mathrm{E}-3 \\
1.206 \mathrm{E}-3 \\
0.000 \mathrm{E}-0 \\
-1.528 \mathrm{E}-4 \\
-9.450 \mathrm{E}-5\end{array}$ & $\begin{array}{r}-1.483 \mathrm{E}-2 \\
-1.191 \mathrm{E}-2 \\
-8.270 \mathrm{E}-3 \\
-4.360 \mathrm{E}-3 \\
-2.173 \mathrm{E}-3 \\
-9.471 \mathrm{E}-4 \\
-3.942 \mathrm{E}-4 \\
0.000 \mathrm{E}-0 \\
5.386 \mathrm{E}-5 \\
3.410 \mathrm{E}-5\end{array}$ & $\begin{array}{l}3.33 \mathrm{E} \\
3.09 \mathrm{E} \\
2.34 \mathrm{E} \\
4.37 \mathrm{E} \\
2.57 \mathrm{E} \\
4.48 \mathrm{E} \\
3.17 \mathrm{E} \\
0.00 \mathrm{E} \\
5.20 \mathrm{E} \\
8.27 \mathrm{E}\end{array}$ & $\begin{array}{l}0 \\
0 \\
0 \\
0 \\
0 \\
0 \\
0 \\
0 \\
0 \\
0\end{array}$ & $\begin{array}{l}8.18 \mathrm{E} \\
7.62 \mathrm{E} \\
8.69 \mathrm{E} \\
9.64 \mathrm{E} \\
1.17 \mathrm{E} \\
9.61 \mathrm{E} \\
1.89 \mathrm{E} \\
0.00 \mathrm{E} \\
3.84 \mathrm{E} \\
2.94 \mathrm{E}\end{array}$ & $\begin{array}{l}0 \\
0 \\
0 \\
0 \\
1 \\
0 \\
1 \\
0 \\
1 \\
1\end{array}$ & $\begin{array}{l}1.35 \mathrm{E} \\
1.56 \mathrm{E} \\
2.05 \mathrm{E} \\
1.95 \mathrm{E} \\
2.96 \mathrm{E} \\
2.02 \mathrm{E} \\
2.64 \mathrm{E} \\
0.00 \mathrm{E} \\
6.70 \mathrm{E} \\
6.57 \mathrm{E}\end{array}$ & $\begin{array}{l}1 \\
1 \\
1 \\
1 \\
1 \\
1 \\
1 \\
1 \\
1 \\
1 \\
1\end{array}$ & $\begin{array}{l}192.61 \\
182.03 \\
200.41 \\
198.55 \\
198.58 \\
210.96 \\
212.64 \\
204.24 \\
254.96 \\
205.51\end{array}$ & $\begin{array}{l}5.08 \\
3.39 \\
3.54 \\
4.33 \\
4.55 \\
5.03 \\
5.21 \\
3.76 \\
4.25 \\
6.52\end{array}$ \\
\hline $\begin{array}{l}700 \\
720 \\
740\end{array}$ & $\begin{array}{l}1.146 \mathrm{E}-1 \\
2.511 \mathrm{E}-2 \\
5.347 \mathrm{E}-3\end{array}$ & $\begin{array}{l}-5.977 \mathrm{E}-5 \\
-2.430 \mathrm{E}-5 \\
-2.173 \mathrm{E}-6\end{array}$ & $\begin{array}{l}1.249 \mathrm{E}-5 \\
3.262 \mathrm{E}-6 \\
1.181 \mathrm{E}-6\end{array}$ & $\begin{array}{l}2.63 \mathrm{E} \\
6.38 \mathrm{E} \\
6.34 \mathrm{E}\end{array}$ & $\begin{array}{l}0 \\
0 \\
0\end{array}$ & $\begin{array}{l}2.52 \mathrm{E} \\
1.39 \mathrm{E} \\
3.26 \mathrm{E}\end{array}$ & $\begin{array}{l}1 \\
2 \\
1\end{array}$ & $\begin{array}{l}7.96 \mathrm{E} \\
5.49 \mathrm{E} \\
4.58 \mathrm{E}\end{array}$ & $\begin{array}{l}1 \\
1 \\
1\end{array}$ & $\begin{array}{r}194.79 \\
157.16 \\
75.46\end{array}$ & $\begin{array}{l}3.09 \\
9.37 \\
4.59\end{array}$ \\
\hline
\end{tabular}


Table 5 Relative deviations (\%) of the over-all mean color matching functions by the method of adjustment from those by the method of constant stimuli.

\begin{tabular}{|c|c|c|c|c|}
\hline$\lambda(\mathrm{nm})$ & $\Delta \bar{r}(\lambda)$ & & $\Delta \bar{g}(\lambda)$ & $\Delta \vec{b}(\lambda)$ \\
\hline 395 & $1.34 \mathrm{E} \quad 1$ & 1 & $1.62 \mathrm{E} \quad 1$ & $-6.71 E-1$ \\
\hline $\begin{array}{l}400 \\
410 \\
420 \\
430 \\
440 \\
450 \\
460 \\
470 \\
480 \\
490\end{array}$ & $\begin{array}{rr}-3.43 \mathrm{E} & 0 \\
1.23 \mathrm{E} & 1 \\
1.15 \mathrm{E} & 0 \\
2.19 \mathrm{E} & 0 \\
-1.48 \mathrm{E} & 0 \\
3.42 \mathrm{E} & -2 \\
0.00 \mathrm{E} & 0 \\
-1.18 \mathrm{E} & 0 \\
-1.46 \mathrm{E} & 0 \\
-1.37 \mathrm{E} & 0\end{array}$ & $\begin{array}{l}0 \\
1 \\
0 \\
0 \\
0 \\
2 \\
0 \\
0 \\
0 \\
0\end{array}$ & $\begin{array}{rr}3.28 \mathrm{E} & 0 \\
1.11 \mathrm{E} & 1 \\
1.03 \mathrm{E}-1 \\
2.68 \mathrm{E} \quad 0 \\
-2.53 \mathrm{E}-1 \\
1.43 \mathrm{E} & 0 \\
0.00 \mathrm{E} & 0 \\
1.97 \mathrm{E} & 0 \\
6.79 \mathrm{E}-1 \\
1.10 \mathrm{E}-3\end{array}$ & $\begin{array}{rr}-2.35 \mathrm{E} & 0 \\
4.08 \mathrm{E}-1 \\
-4.47 \mathrm{E} & 0 \\
-2.70 \mathrm{E} & 0 \\
-5.59 \mathrm{E} & 0 \\
-4.10 \mathrm{E} & 0 \\
0.00 \mathrm{E} & 0 \\
2.32 \mathrm{E}-1 \\
-2.96 \mathrm{E}-1 \\
-1.12 \mathrm{E} & 0\end{array}$ \\
\hline $\begin{array}{l}500 \\
510 \\
520 \\
530 \\
540 \\
550 \\
560 \\
570 \\
580 \\
590\end{array}$ & $\begin{array}{rr}-1.43 \mathrm{E} & 0 \\
-1.05 \mathrm{E} & 0 \\
-1.81 \mathrm{E} & 0 \\
0.00 \mathrm{E} & 0 \\
-3.78 \mathrm{E} & 0 \\
-2.13 \mathrm{E} & 0 \\
-2.23 \mathrm{E} & 0 \\
2.38 \mathrm{E} & -1 \\
-1.42 \mathrm{E} & 0 \\
-1.33 \mathrm{E} & 0\end{array}$ & $\begin{array}{l}0 \\
0 \\
0 \\
0 \\
0 \\
0 \\
0 \\
1 \\
0 \\
0\end{array}$ & $\begin{array}{r}1.85 \mathrm{E} \quad 0 \\
9.87 \mathrm{E}-1 \\
9.64 \mathrm{E}-1 \\
0.00 \mathrm{E} \quad 0 \\
-6.09 \mathrm{E}-1 \\
-9.77 \mathrm{E}-1 \\
-9.63 \mathrm{E}-1 \\
-2.45 \mathrm{E} \quad 0 \\
-1.62 \mathrm{E} \quad 0 \\
-1.62 \mathrm{E} \quad 0\end{array}$ & $\begin{array}{rr}-1.36 \mathrm{E} & -1 \\
-4.90 \mathrm{E} & 0 \\
-1.20 \mathrm{E} & 1 \\
0.00 \mathrm{E} & 0 \\
1.75 \mathrm{E} & 1 \\
1.25 \mathrm{E} & 1 \\
9.64 \mathrm{E} & 0 \\
9.81 \mathrm{E} & 0 \\
9.32 \mathrm{E} & 0 \\
6.76 \mathrm{E} & 0\end{array}$ \\
\hline $\begin{array}{l}600 \\
610 \\
620 \\
630 \\
640 \\
650 \\
660 \\
670 \\
680 \\
690\end{array}$ & $\begin{array}{rr}-1.32 \mathrm{E} & 0 \\
-1.13 \mathrm{E} & 0 \\
-6.59 \mathrm{E}-1 \\
-2.04 \mathrm{E} & 0 \\
-1.19 \mathrm{E} & 0 \\
-1.82 \mathrm{E} & 0 \\
-8.71 \mathrm{E} & -1 \\
0.00 \mathrm{E} & 0 \\
-1.42 \mathrm{E} & 0 \\
-3.48 \mathrm{E} & 0\end{array}$ & $\begin{array}{l}0 \\
0 \\
1 \\
0 \\
0 \\
0 \\
1 \\
0 \\
0 \\
0\end{array}$ & $\begin{array}{rr}-9.88 \mathrm{E}-1 \\
-2.60 \mathrm{E} & 0 \\
-3.11 \mathrm{E} & 0 \\
-2.16 \mathrm{E} & 0 \\
1.90 \mathrm{E} & 0 \\
-3.92 \mathrm{E} & 0 \\
-1.14 \mathrm{E} & 0 \\
0.00 \mathrm{E} & 0 \\
-1.80 \mathrm{E} & 0 \\
2.56 \mathrm{E} & -1\end{array}$ & $\begin{array}{rc}4.40 \mathrm{E} & 0 \\
6.89 \mathrm{E} & 0 \\
1.86 \mathrm{E} & 0 \\
9.55 \mathrm{E} & 0 \\
1.61 \mathrm{E} & 0 \\
7.93 \mathrm{E} & 0 \\
-6.35 \mathrm{E} & 0 \\
0.00 \mathrm{E} & 0 \\
-4.90 \mathrm{E} & 1 \\
-3.59 \mathrm{E} & 1\end{array}$ \\
\hline $\begin{array}{l}700 \\
720 \\
740\end{array}$ & $\begin{array}{ll}-4.15 \mathrm{E} & 0 \\
-5.59 \mathrm{E} & 0 \\
-4.39 \mathrm{E} & 0\end{array}$ & $\begin{array}{l}0 \\
0 \\
0\end{array}$ & $\begin{array}{ll}3.88 \mathrm{E} & 0 \\
1.64 \mathrm{E} & 0 \\
4.30 \mathrm{E} & 0\end{array}$ & $\begin{array}{rr}1.79 \mathrm{E} & 1 \\
-4.98 \mathrm{E} & 0 \\
3.75 \mathrm{E} & 1\end{array}$ \\
\hline
\end{tabular}

Table 6 Relative deviations (\%) of the over-all mean color matching functions by the method of constant stimuli from the CIE color matching functions.

\begin{tabular}{|c|c|c|c|c|c|}
\hline$\lambda(\mathrm{nm})$ & $\Delta \vec{r}(\lambda)$ & $\Delta \bar{g}(\lambda)$ & & $\Delta \bar{b}(\lambda)$ & \\
\hline 395 & $-5.07 \mathrm{E} \quad 1$ & $-4.62 \mathrm{E}$ & 1 & $3.30 \mathrm{E}$ & 0 \\
\hline $\begin{array}{l}400 \\
410 \\
420 \\
430 \\
440 \\
450 \\
460 \\
470 \\
480 \\
490\end{array}$ & $\begin{array}{rr}-4.47 \mathrm{E} & 1 \\
-5.25 \mathrm{E} & 1 \\
-4.92 \mathrm{E} & 1 \\
-4.24 \mathrm{E} & 1 \\
-3.35 \mathrm{E} & 1 \\
-3.46 \mathrm{E} & 1 \\
0.00 \mathrm{E} & 0 \\
-2.11 \mathrm{E} & 1 \\
-1.26 \mathrm{E} & 1 \\
-8.97 \mathrm{E} & 0\end{array}$ & $\begin{array}{r}-2.61 \mathrm{E} \\
-3.34 \mathrm{E} \\
-3.50 \mathrm{E} \\
-3.14 \mathrm{E} \\
-1.69 \mathrm{E} \\
-2.88 \mathrm{E} \\
0.00 \mathrm{E} \\
-1.23 \mathrm{E} \\
-1.02 \mathrm{E} \\
-6.16 \mathrm{E}\end{array}$ & $\begin{array}{l}1 \\
1 \\
1 \\
1 \\
1 \\
1 \\
0 \\
1 \\
1 \\
0\end{array}$ & $\begin{aligned} & 5.56 \mathrm{E} \\
&- 8.55 \mathrm{E} \\
&-1.49 \mathrm{E} \\
&-1.06 \mathrm{E} \\
& 6.41 \mathrm{E} \\
& 1.41 \mathrm{E}- \\
& 0.00 \mathrm{E} \\
&-1.94 \mathrm{E} \\
&-1.52 \mathrm{E}- \\
& 3.06 \mathrm{E}\end{aligned}$ & $\begin{array}{r}0 \\
0 \\
1 \\
1 \\
0 \\
-1 \\
0 \\
0 \\
-1 \\
0\end{array}$ \\
\hline $\begin{array}{l}500 \\
510 \\
520 \\
530 \\
540 \\
550 \\
560 \\
570 \\
580 \\
590\end{array}$ & $\begin{array}{rl}-1.15 \mathrm{E} & 1 \\
-5.61 \mathrm{E} & 0 \\
-2.59 \mathrm{E} & 0 \\
0.00 \mathrm{E} & 0 \\
-1.81 \mathrm{E} & 1 \\
-1.62 \mathrm{E} & 1 \\
-1.33 \mathrm{E} & 1 \\
-1.55 \mathrm{E} & 1 \\
-1.27 \mathrm{E} & 1 \\
-1.26 \mathrm{E} & 1\end{array}$ & $\begin{array}{r}-5.17 \mathrm{E} \\
-3.07 \mathrm{E} \\
-3.12 \mathrm{E} \\
0.00 \mathrm{E} \\
1.20 \mathrm{E} \\
2.55 \mathrm{E} \\
3.50 \mathrm{E} \\
6.09 \mathrm{E} \\
8.79 \mathrm{E} \\
8.28 \mathrm{E}\end{array}$ & $\begin{array}{l}0 \\
0 \\
0 \\
0 \\
0 \\
0 \\
0 \\
0 \\
0 \\
0\end{array}$ & $\begin{array}{r}8.46 \mathrm{E} \\
1.61 \mathrm{E} \\
4.26 \mathrm{E} \\
0.00 \mathrm{E} \\
-4.00 \mathrm{E} \\
-2.60 \mathrm{E} \\
-1.66 \mathrm{E} \\
5.64 \mathrm{E} \\
4.17 \mathrm{E} \\
9.98 \mathrm{E}\end{array}$ & $\begin{array}{l}0 \\
1 \\
1 \\
0 \\
1 \\
1 \\
1 \\
0 \\
1 \\
1\end{array}$ \\
\hline $\begin{array}{l}600 \\
610 \\
620 \\
630 \\
640 \\
650 \\
660 \\
670 \\
680 \\
690\end{array}$ & $\begin{array}{lr}-1.09 \mathrm{E} & 1 \\
-1.00 \mathrm{E} & 1 \\
-7.78 \mathrm{E} & 0 \\
-3.15 \mathrm{E} & 0 \\
-8.04 \mathrm{E} & -1 \\
2.64 \mathrm{E} & 0 \\
2.05 \mathrm{E} & 0 \\
0.00 \mathrm{E} & 0 \\
5.58 \mathrm{E} & 0 \\
7.70 \mathrm{E} & 0\end{array}$ & $\begin{array}{l}6.34 \mathrm{E} \\
9.12 \mathrm{E} \\
1.57 \mathrm{E} \\
3.73 \mathrm{E} \\
8.32 \mathrm{E} \\
6.50 \mathrm{E} \\
6.60 \mathrm{E} \\
0.00 \mathrm{E} \\
4.96 \mathrm{E} \\
3.41 \mathrm{E}\end{array}$ & $\begin{array}{l}0 \\
0 \\
1 \\
0 \\
0 \\
1 \\
1 \\
0 \\
1 \\
1\end{array}$ & $\begin{array}{l}1.92 \mathrm{E} \\
3.01 \mathrm{E} \\
4.78 \mathrm{E} \\
4.93 \mathrm{E} \\
7.77 \mathrm{E} \\
1.40 \mathrm{E} \\
3.18 \mathrm{E} \\
0.00 \mathrm{E} \\
5.72 \mathrm{E} \\
4.24 \mathrm{E}\end{array}$ & $\begin{array}{l}2 \\
2 \\
2 \\
2 \\
2 \\
3 \\
3 \\
0 \\
3 \\
3\end{array}$ \\
\hline $\begin{array}{l}700 \\
720 \\
740\end{array}$ & $\begin{array}{rr}1.35 \mathrm{E} & 0 \\
-6.90 \mathrm{E} & -1 \\
-1.06 \mathrm{E} & 1\end{array}$ & $\begin{array}{l}4.98 \mathrm{E} \\
2.77 \mathrm{E} \\
8.54 \mathrm{E}\end{array}$ & $\begin{array}{l}1 \\
2 \\
2\end{array}$ & $\begin{array}{l}1.48 \mathrm{E} \\
3.01 \mathrm{E} \\
2.25 \mathrm{E}\end{array}$ & $\begin{array}{l}3 \\
3 \\
4\end{array}$ \\
\hline
\end{tabular}

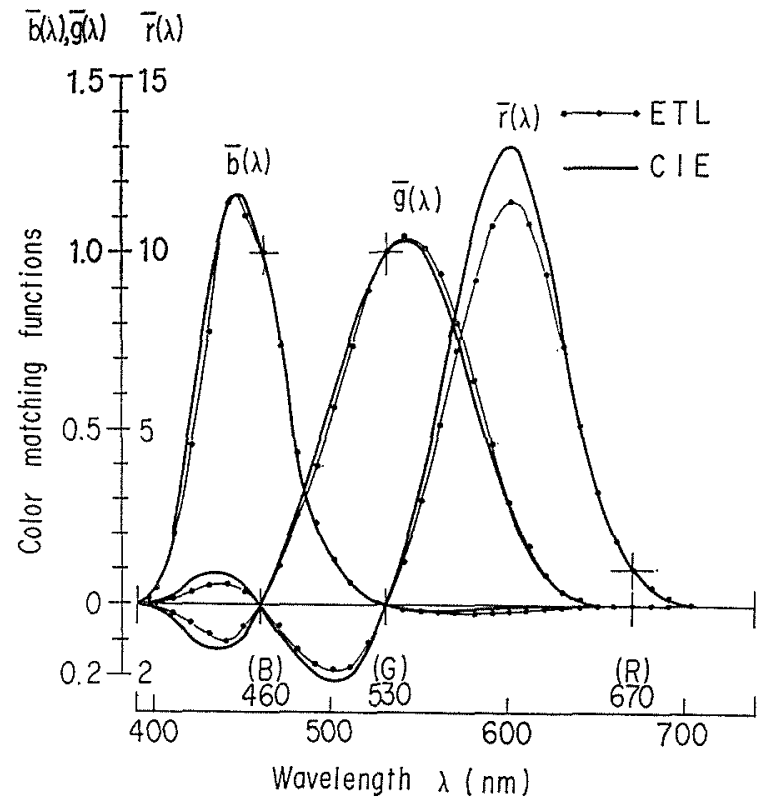

Fig. 4 Comparison of the ETL $10^{\circ}$ color matching functions (thin lines with dots) with the CIE 1964 color matching functions (thick lines) transformed to the same trichromatic system as the ETL data are expressed.

Each set of the color matching functions is so normalized that the respective functions have the value unity at the wavelength of the corresponding reference stimulus.

data are tabulated in Table 6 .

Both of the two ETL mean data showed a little difference from the CIE data in the region of long wavelengths in the visible range as shown in Fig. 4, where the CIE data were transformed to the same trichromatic system as the ETL data were expressed.

\subsection{Effect of field intensity}

In order to explain the discrepancy between the CIE data and the ETL data in the long wavelength region, the effect of field intensity on the color matching functions was examined. Color matching functions at four levels of intensity 200, 500, 1,000 and 2,000 trolands were determined by the method of adjustment for an observer (one of the authors, K.K.). Measurements were made from 530 to $670 \mathrm{~nm}$ at every $10 \mathrm{~nm}$.

Fig. 5 shows the color matching functions determined by K.K. at four different levels of intensity and the CIE ones transformed to the same RGB system on which the ETL color matching functions were determined. In Fig. 5(A), as the field intensity increases from 200 to 2000 trd $(d \rightarrow a)$, the $r(\lambda)$ function here obtained has larger values and gets nearer to the CIE curve $(e)$, while the $\bar{g}(\lambda)$ function shows no remarkable changes. Vertical bars on the $\bar{g}(\lambda)$ curve show ranges of variation by the intensity changes from 200 to 2000 trd.

In Fig. 5(B), the $\vec{b}(\lambda)$ function also changes as 


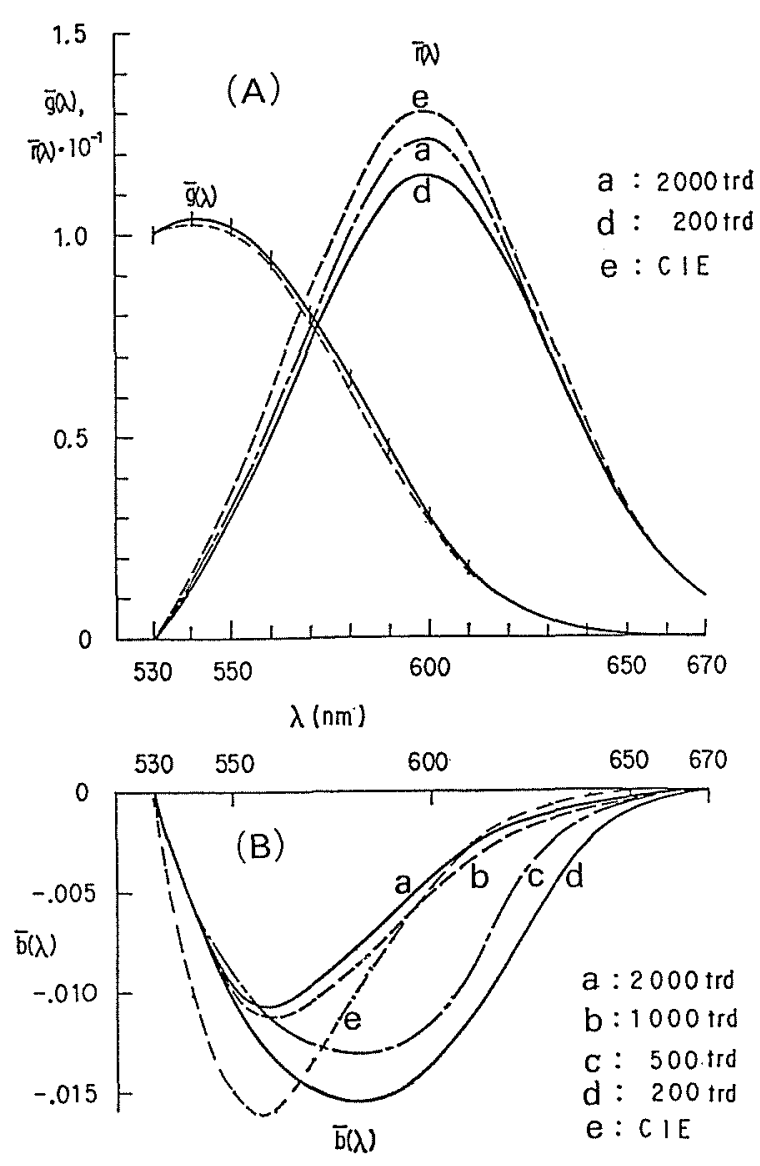

Fig. 5 Effect of field intensity on the color matching functions of an observer ( $K$. $K$.)

$(A)$ : The $\vec{r}(\lambda)$ function is shown by two curves $(a, d)$ at two intensity levels of 200 and $2000 \mathrm{trd}$. The curves at the levels of 500 and 1000 trd are not drawn for clarification, although they are to be drawn between the two curves $(a, d)$. Scale of the ordinate for $\bar{r}(\lambda)$ is reduced to the tenth of that for $\bar{g}(\lambda)$. $(B)$ : Scale of the ordinate for $\bar{b}(\lambda)$ is enlarged to ten times of that for $\bar{g}(\lambda)$.

field intensity increases, and the $\bar{b}(\lambda)$ curves $(a, b$, c, d) show a slight difference from the CIE $\bar{b}(\lambda)$ curve (e) in their shapes. These changes of the $\bar{b}(\lambda)$ function have large effect on color matching properties, but few contribution to the spectral luminous efficiency function.7)

The results suggest that the discrepancy between the ETL color matching functions and the CIE ones might originate from the fact that they were determined under the different conditions. Stiles' experiments were made at higher intensity level in the long wavelength region, considering the effect of rod intrusion, on the other hand, the ETL color matching data were determined under a constant level of intensity of about 200 trolands.

The further studies of the field intensity effect and the chromatic adaptation effect on the color matching functions have remained in future.

\section{Conclusion}

(a) Additivity law in the color equation held good for the $10^{\circ}$ field as well as the $2^{\circ}$ field.

(b) It was suggested that color matching properties for the $10^{\circ}$ field depended on the kinds of the chromatic stimuli for the pre-adaptation.

(c) Two sets of the indivisual color matching functions determined by MCS and MA showed obvious differences in respect to the all observers, in comparison with the coefficients of deviation among the measured values by the respective observers.

(d) There were, however, no significant differences between the two sets of the over-all mean color matching functions, which were obtained by averaging those two sets of the indivisual color matching functions.

(e) Both of the two ETL data by MCA and MA showed a little difference from the CIE data in the long wavelength region.

(f) As the field intensity increases from 200 to $2,000 \mathrm{trd}$, the discrepancy between the ETL data and the CIE data became smaller.

\section{Acknowledgements}

We would like to express our deep appreciation to Dr. W. S. Stiles of England and Dr. Gunter Wyszecki of the National Research Council of Canada for their kindness giving us the blue copies of the NPL trichromator when we intended to construct the ETL trichromator used in this study.

We would like to thank Dr. N. Ooba for his valuable comments and criticism of the original manuscript.

We are also grateful to the members of our Applied Optics Section, ETL, for their kind cooperation as the observers of the present study, and to the members of the ETL computing center for their cooperation in the data processing.

We also wish to record that the engineers of Nippon Kogaku (NIKON) Co., Ltd. were associated with the construction of the ETL trichromator.

\section{References}

(1) K. Katori, M. Fuwa and Y. Sugiyama: Researches on color matching functions, Res. Electrotech. Lab. No. 773 (1977) 1 110 (in Japanese).

(2) W. D. Wright: A re-determination of the trichromatic coefficients of the spectral colours: Trans. Opt. Soc. 30 (1928 1929) 141 164.

(3) J. Guild : The colorimetric properties of the spectrum, Phil. Trans. Roy Soc. (London) A230 (1931) $149 \sim 187$.

(4) W. S. Stiles and J. M. Burch: N.P.L. color-matching in vestigation: Final report (1958): Optica Acta 6 (1959) 1 26.

(5) N.I. Speranskaya: Determination of spectrum color coordinates for twenty-seven normal observers, Optics and Spectroscopy 7 (1959) $424 \sim 428$.

(6) W. S. Stlles: The basic data of colour matching, Phys. Soc. Year Book (1955) 44 65. 
(7) K. Katori and M. Fuwa : Spectral luminous efficiency function derived from color matching functions of 10 degree field, Acta Chromatica 3 (1979) $129 \sim 140$.

(8) P.W. Trezona : Additivity of colour equations, Proc. Phys. Soc. (London) B66 (1953) 548 556.

(9) W. S. Stiles: N.P.L. colour matching investigation: Addendum on additivity, Optica Acta 10 (1964) $229 \sim 232$

(10) F. J. J. Clarke : Extra-foveal vision and the additivity concept, Farbe 11 (1962) 19 28. (ii) R. D. Lozano and P.A. Palmer : The additivity of large-field colour matching functions, Vision Res. 7 (1967) $929 \sim 937$.

(12) B. H. Crawford : Colour matching and adaptation, Vision Res. 5 (1965) $71 \sim 78$.

(13) G. Wyszecki : Color matching at moderate to high levels of retinal illuminance: a pilot study, Vision Res. 18 (1978) $341 \sim 346$.

Received 29 Oct. 1980; Revision Received 7 Jan. 1981 